\title{
THE SUMMATION OF SERIES OF ORTHOGONAL POLYNOMIALS*
}

\author{
BY DUNHAM JACKSON
}

1. Introduction. The convergence of series of orthogonal polynomials can be treated under very broad hypotheses as to the character of the weight function with respect to which the property of orthogonality holds, if compensating restrictions are placed on the function whose series development is under consideration. $\uparrow \mathrm{It}$ is to be shown here that certain questions of summability lend themselves to a comparable treatment, simple as to demonstration and in some ways perhaps crude as to results, but characterized by broad generality in the hypotheses on the weight function. The discussion of summability, unlike the particular theory of convergence to which reference has been made, depends on the representation of the partial sum of the series by means of the Christoffel-Darboux identity. The method will be illustrated first under simple conditions, which will then be seen to admit generalization in various directions.

The problem of trigonometric approximation, as usual, is similar in principle but somewhat different in detail.

2. Application of Schwarz's Inequality to the First Arithmetic Mean. Let $p_{0}(x), p_{1}(x), \cdots$ be the system of normalized orthogonal polynomials for the interval $(-1,1)$ corresponding to the summable non-negative weight function $\rho(x)$, let a function $f(x)$ (always supposed to be such that $\rho f$ is summable, and to be subjected to further restrictions later) be expanded in series of these polynomials, and let $s_{n}(x)$ be the partial sum of the series through terms of the $n$th degree. Let

$$
\sigma_{n}(x)=\frac{1}{n} \sum_{k=0}^{n-1} s_{k}(x) .
$$

The sums $s_{k}(x)$ and the corresponding errors of approximation $s_{k}(x)-f(x)$ can be represented in compact form by use of the

* Presented to the Society, April 7, 1934.

$\dagger$ See, for example, the writer's paper entitled Certain problems of closest approximation, this Bulletin, vol. 39 (1933), pp. 889-906. 
Christoffel-Darboux identity, ${ }^{*}$ and this representation gives for the error of the arithmetic mean the expression

$$
\begin{aligned}
\sigma_{n}(x)-f(x) & =\frac{1}{n} \int_{-1}^{1} \rho(t)[f(t)-f(x)] \\
& \times \sum_{k=0}^{n-1} \frac{a_{k}}{a_{k+1}} \cdot \frac{p_{k+1}(t) p_{k}(x)-p_{k}(t) p_{k+1}(x)}{t-x} d t
\end{aligned}
$$

where $a_{k}>0$ is the leading coefficient in the normalized polynomial $p_{k}(x)$. Let the difference quotient $[f(t)-f(x)] /(t-x)$, for any specified value of $x$, be denoted by $\phi(t)$, and let

$$
H_{n}(x, t)=\sum_{k=0}^{n-1} \frac{a_{k}}{a_{k+1}} p_{k+1}(t) p_{k}(x) .
$$

Then, if $\rho \phi$ is summable,

$$
\begin{aligned}
n\left[\sigma_{n}(x)-f(x)\right]= & \int_{-1}^{1} \rho(t) \phi(t) H_{n}(x, t) d t \\
& -\int_{-1}^{1} \rho(t) \phi(t) H_{n}(t, x) d t .
\end{aligned}
$$

Under the assumption that $\rho(t)[\phi(t)]^{2}$ is summable, let Schwarz's inequality be applied to the first integral on the right, the integrand being regarded as the product of the factors $[\rho(t)]^{1 / 2} \phi(t)$ and $[\rho(t)]^{1 / 2} H_{n}(x, t)$. It appears that

$$
\begin{aligned}
& {\left[\int_{-1}^{1} \rho(t) \phi(t) H_{n}(x, t) d t\right]^{2}} \\
& \quad \leqq \int_{-1}^{1} \rho(t)\lfloor\phi(t)]^{2} d t \int_{-1}^{1} \rho(t)\left[H_{n}(x, t)\right]^{2} d t .
\end{aligned}
$$

By the property of orthogonality of the $p$ 's,

$$
\int_{-1}^{1} \rho(t)\left[H_{n}(x, t)\right]^{2} d t=\sum_{k=0}^{n-1}\left[\left(a_{k} / a_{k+1}\right) p_{k}(x)\right]^{2} .
$$

It is known $\dagger$ that $a_{k} / a_{k+1}<1$, and hence, in terms of the notation

* See, for example, D. Jackson, Series of orthogonal polynomials, Annals of Mathematics, (2), vol. 34 (1933), pp. 527-545, p. 531.

$\dagger$ See Annals of Mathematics, loc. cit., pp. 531-532. 


$$
\begin{gathered}
K_{n}(x, t)=p_{0}(x) p_{0}(t)+p_{1}(x) p_{1}(t)+\cdots+p_{n}(x) p_{n}(t), \\
\int_{-1}^{1} \rho(t)\left[H_{n}(x, t)\right]^{2} d t \leqq K_{n}(x, x) .
\end{gathered}
$$

By the property of orthogonality once more,

$$
\int_{-1}^{1} \rho(t)\left[K_{n}(x, t)\right]^{2} d t=\sum_{k=0}^{n}\left[p_{k}(x)\right]^{2}=K_{n}(x, x) .
$$

If $\rho(t)$ has a positive lower bound, say $\rho(t) \geqq v>0$ for $-1 \leqq t \leqq 1$,

(4) $\quad \int_{-1}^{1}\left[K_{n}(x, t)\right]^{2} d t \leqq \frac{1}{v} \int_{-1}^{1} \rho(t)\left[K_{n}(x, t)\right]^{2} d t=\frac{1}{v} K_{n}(x, x)$.

A lemma* giving a relation of inequality between an integral involving a polynomial and the maximum of the absolute value of the polynomial can be stated in a special case as follows. If $P_{n}(t)$ is a polynomial of the nth degree, and if

$$
H_{n 2}=\int_{-1}^{1}\left[P_{n}(t)\right]^{2} d t
$$

then

$$
\left|P_{n}(t)\right| \leqq \frac{2\left[2(n+1) H_{n 2}\right]^{1 / 2}}{\left(1-t^{2}\right)^{1 / 2}}
$$

for $-1<t<1$. As $K_{n}(x, t)$ is for any fixed value of $x$ a polynomial of the $n$th degree in $t$, this lemma may be combined with the relation (4) to give

$$
\left|K_{n}(x, t)\right| \leqq \frac{2\left[2(n+1)(1 / v) K_{n}(x, x)\right]^{1 / 2}}{\left(1-t^{2}\right)^{1 / 2}}
$$

and in particular $\dagger$

$$
K_{n}(x, x) \leqq \frac{2\left[(2 / v)(n+1) K_{n}(x, x)\right]^{1 / 2}}{\left(1-x^{2}\right)^{1 / 2}},
$$

* See this Bulletin, loc. cit., p. 897, Lemma 4.

$\dagger$ See also J. A. Shohat, On the development of continuous functions in series of Tchebycheff polynomials, Transactions of this Society, vol. 27 (1925), pp. 537-550; G. Szegö, Über den asymptotischen Ausdruck von Polynomen, die durch eine Orthogonalitätseigenschaft definiert sind, Mathematische Annalen, vol. 86 (1922), pp. 114-139, p. 129. 
(5) $\left[K_{n}(x, x)\right]^{1 / 2} \leqq \frac{2[(2 / v)(n+1)]^{1 / 2}}{\left(1-x^{2}\right)^{1 / 2}}, \quad K_{n}(x, x) \leqq \frac{8(n+1)}{v\left(1-x^{2}\right)}$.

Hence, in view of (2) and (3), the first integral in the righthand member of (1) does not exceed a constant multiple of $n^{1 / 2}$. Similar analysis applies to the second integral, since

$$
\int_{-1}^{1} \rho(t)\left[H_{n}(t, x)\right]^{2} d t=\sum_{k=0}^{n-1}\left[\left(a_{k} / a_{k+1}\right) p_{k+1}(x)\right]^{2} \leqq K_{n}(x, x) .
$$

If $\rho \phi^{2}$ is summable, therefore, while $\rho$ has a positive lower bound, $\sigma_{n}(x)$ converges toward $f(x)$ as $n$ becomes infinite, $x$ being interior to the interval $(-1,1)$, and $\left|\sigma_{n}(x)-f(x)\right|$ does not exceed a quantity of the order of $n^{-1 / 2}$. If $\int \rho \phi^{2}$ as a function of $x$ is bounded over a set of points contained in a closed interval interior to $(-1,1)$, the convergence is uniform over that point set.

A sufficient condition* for convergence of $s_{n}(x)$ toward $f(x)$ in the interior of the interval is that $\lim _{\delta \rightarrow 0} \omega(\delta) / \delta^{1 / 2}=0$, where $\omega(\delta)$ is the modulus of continuity of $f(x)$ for $-1 \leqq x \leqq 1$. A first comparison shows the new condition for convergence of $\sigma_{n}(x)$ in its most unfavorable light; for convergence of $s_{n}(x)$ of course implies convergence of $\sigma_{n}(x)$, and the hypothesis that $\omega(\delta) / \delta^{1 / 2} \rightarrow 0$ does not require that $\rho \phi^{2}$ be summable, even if $\rho$ itself is bounded. The following points are to be emphasized, however, on behalf of the new method.

(a) The theorem on convergence of $s_{n}(x)$ does not give the information that has been obtained by the new method as to the order of magnitude of $\sigma_{n}(x)-f(x)$.

(b) The hypothesis on $\omega(\delta)$ demands that $f(x)$ be continuous and subject to further restriction throughout the entire interval $\uparrow(-1,1)$, while the summability of $\rho \phi^{2}$ for a particular $x$ does not require that $f(x)$ be continuous or even bounded elsewhere.

(c) For mere convergence of $\sigma_{n}(x)$, apart from degree of convergence, summability of $\rho \phi^{2}$ can readily be replaced by a less stringent requirement, as will be shown in the next section.

* This Bulletin, loc. cit., p. 906, Theorem 15.

$\dagger$ It is obvious, but irrelevant for the comparison, that for a more general form of statement the definition of $f(x)$ at a set of points of measure zero is immaterial. 
(d) The convergence theorem can also be generalized in other ways, as will be pointed out later.

3. More General Function $f(x)$. Let it be supposed now that $\rho(t)|\phi(t)|^{\alpha}$ is summable, where $\alpha$ has a value belonging to the range $1<\alpha<2$. With reference to the first integral on the right of (1) once more, let Hölder's inequality be applied to the product of the factors $[\rho(t)]^{1 / \alpha}|\phi(t)|$ and $[\rho(t)]^{(\alpha-1) / \alpha}\left|H_{n}(x, t)\right|$ :

$$
\begin{aligned}
& \left|\int_{-1}^{1} \rho(t) \phi(t) H_{n}(x, t) d t\right| \leqq \int_{-1}^{1} \rho(t)\left|\phi(t) H_{n}(x, t)\right| d t \\
& \leqq\left[\int_{-1}^{1} \rho(t)|\phi(t)| \alpha d t\right]^{1 / \alpha}\left[\int_{-1}^{1} \rho(t)\left|H_{n}(x, t)\right| \alpha /(\alpha-1) d t\right]^{(\alpha-1) / \alpha} .
\end{aligned}
$$

Let it be assumed still that $\rho(t)$ has a lower bound $v>0$. Let

$$
h_{n 2}=\int_{-1}^{1} \rho(t)\left[H_{n}(x, t)\right]^{2} d t .
$$

It has been shown above, in (3) and (5), that $h_{n 2}$ does not exceed a constant multiple of $n$, or in symbols

$$
h_{n 2}=O(n)
$$

this relation holding uniformly for values of $x$ belonging to any closed interval interior to $(-1,1)$. The integral of $H_{n}^{2}$ from -1 to 1 does not exceed $h_{n 2} / v$, and so, by a lemma analogous to one already cited, but giving an upper bound for the absolute value of the polynomial in the integrand throughout the whole closed interval of integration, *

$$
\left|H_{n}(x, t)\right|=O\left\lfloor n\left(h_{n 2}\right)^{1 / 2}\right\rfloor
$$

uniformly for $-1 \leqq t \leqq 1$ and $-1 \leqq x \leqq 1$. From this and (8) it appears that $\left|H_{n}(x, t)\right|=O\left(n^{3 / 2}\right)$, uniformly for $t$ in $(-1,1)$ and for $x$ in any closed interval interior to $(-1,1)$.

For a specified $x$, let $\mu_{n}$ be the maximum of $\left|H_{n}(x, t)\right|$ in $(-1,1)$. If $\gamma>2$,

* See this Bulletin, loc. cit., pp. 893-894, Lemma 2. It is to be understood in the statement of the Lemma that $n \geqq 1$. 


$$
\begin{aligned}
\int_{-1}^{1} \rho(t)\left|H_{n}(x, t)\right|^{\gamma} d t & =\int_{-1}^{1}\left|H_{n}(x, t)\right|^{\gamma-2} \rho(t)\left[H_{n}(x, t)\right]^{2} d t \\
& \leqq \mu_{n}^{\gamma-2} \int_{-1}^{1} \rho(t)\left[H_{n}(x, t)\right]^{2} d t=\mu_{n}^{\gamma-2} h_{n 2} .
\end{aligned}
$$

Since $\mu_{n}=O\left(n^{3 / 2}\right)$ and $h_{n 2}=O(n)$,

$$
\int_{-1}^{1} \rho(t)\left|H_{n}(x, t)\right| \gamma d t=O\left(n^{(3 \gamma / 2)-2}\right) .
$$

Substitution of this in (6), with $\gamma=\alpha /(\alpha-1)$, gives

$$
\left|\int_{-1}^{1} \rho(t) \phi(t) H_{n}(x, t) d t\right|=O\left(n^{(3 / 2)-(2 / \gamma)}\right)=O\left(n^{(2 / \alpha)-(1 / 2)}\right)
$$

the specification that $\alpha<2$ insures that $\gamma>2$. The second integral in (1) can be treated in the same way, and it follows that

$$
\left|\sigma_{n}(x)-f(x)\right|=O\left(n^{(2 / \alpha)-(3 / 2)}\right) .
$$

In any closed interval interior to $(-1,1)$ the relation holds uniformly over any set of values of $x$ for which $\int \rho|\phi|^{\alpha}$ is bounded.

The exponent $(2 / \alpha)-(3 / 2)$ will be negative if $\alpha>4 / 3$. If $\rho(t)$ has a positive lower bound, and if $x$ is an interior point of the interval $(-1,1), \sigma_{n}(x)$ will converge toward $f(x)$ provided that $\rho(t)|\phi(t)|^{\alpha}$ is summable with a value of $\alpha>4 / 3$, the notation $\phi(t)$ representing the difference quotient $[f(t)-f(x)] /(t-x)$. Further details with regard to uniformity of convergence can be supplied from the preceding paragraph.

The condition just stated is manifestly broader than that which required that $\rho \phi^{2}$ be summable. The condition for convergence of $\sigma_{n}(x)$ toward $f(x)$ will be satisfied, as far as the behavior of $f(t)$ in the neighborhood of the point $t=x$ is concerned, if $\rho(t)$ is bounded in the neighborhood of the point, and if

$$
|f(t)-f(x)|=O\left(|t-x|^{\beta}\right)
$$

as $t$ approaches $x$, with a value of $\beta>1 / 4$. For then

$$
|\phi(t)|=O\left(|t-x|^{\beta-1}\right), \quad|\phi(t)|^{\alpha}=O\left(|t-x|^{(\beta-1) \alpha}\right),
$$

and it will be possible to choose a value of $\alpha>4 / 3$ so that $(\beta-1) \alpha>-1$. 
4. More General Weight Function. A new element is introduced into the problem if the greatest lower bound of $\rho$, instead of being positive, is allowed to be zero. The extent of this generalization will be limited for the purposes of the present argument by the assumption that $[\rho(t)]^{-r}$ is summable over $(-1,1)$ with a value of $r>1$.

The change of hypothesis affects the estimate of an upper bound for $K_{n}(x, x)$. Let

$$
s=2 r /(r+1), \quad p=2 / s=1+(1 / r)>1 .
$$

By application of Hölder's inequality to the integral of the product of the factors $[\rho(t)]^{-1 / p}$ and $[\rho(t)]^{1 / p}\left|K_{n}(x, t)\right|^{s}$, it is seen that*

$$
\begin{aligned}
\int_{-1}^{1}\left|K_{n}(x, t)\right|^{s} d t \\
\\
\quad \leqq\left[\int_{-1}^{1}[\rho(t)]^{-r} d t\right]^{1 /(r+1)}\left[\int_{-1}^{1} \rho(t)\left[K_{n}(x, t)\right]^{2} d t\right]^{r /(r+1)} .
\end{aligned}
$$

The next to the last integral is convergent by hypothesis, and is independent of $x$; the value of the last integral is $K_{n}(x, x)$. By a lemma $\nmid$ which has already been used in a special case,

$$
\left|K_{n}(x, t)\right| \leqq \frac{C n^{(r+1) /(2 r)}\left[K_{n}(x, x)\right]^{1 / 2}}{\left(1-t^{2}\right)^{N / 2}}
$$

for $n \geqq 1$ and for $-1<t<1$, where $N$ is the smallest integer $\geqq(r+1) /(2 r)$, and $C$ is independent of $n, x$, and $t$. In particular, for $t=x$,

$$
\left[K_{n}(x, x)\right]^{1 / 2} \leqq \frac{C n^{(r+1) /(2 r)}}{\left(1-x^{2}\right)^{N / 2}}, \quad K_{n}(x, x) \leqq \frac{C^{2} n^{(r+1) / r}}{\left(1-x^{2}\right)^{N}} .
$$

This calculation holds for any value of $r>0$. If $r>1, N=1$, and

$$
K_{n}(x, x) \leqq \frac{C^{2} n^{(r+1) / r}}{1-x^{2}}
$$

In continuation, it is to be supposed again that $\rho(t)|\phi(t)|^{\alpha}$ is summable with a value of $\alpha$ between 1 and 2 , and inquiry is to

* See this Bulletin, loc. cit., p. 892.

$\dagger$ This Bulletin, loc. cit., p. 897, Lemma 4. 
be made as to a value of $\alpha$ which will insure convergence of $\sigma_{n}(x)$ toward $f(x)$ under present conditions. The relation (6) holds as before. Also (3) is still valid, and if $h_{n 2}$ is defined as in (7), the right-hand member of (13) gives an upper bound for $h_{n 2}$. If $H_{n}(x, t) /\left(h_{n 2}\right)^{1 / 2}$ is denoted by $P_{n}(t)$, it can be asserted* that $\left|P_{n}(t)\right|$ has an upper bound of the order of $n^{(r+1) / r}$ for $-1 \leqq t \leqq 1$; inspection of the proof of the proposition referred to will show that

$$
\left|P_{n}(t)\right| \leqq 2^{(3 r+1) /(2 r)} n^{(r+1) / r}\left[\int_{-1}^{1}[\rho(t)]^{-r} d t\right]^{1 /(2 r)}
$$

for $n \geqq 1$, so that $\left|P_{n}(t)\right|=O\left(n^{(r+1) / r}\right)$ uniformly for $-1 \leqq t \leqq 1$, $-1 \leqq x \leqq 1$. Consequently $\left|H_{n}(x, t)\right|=O\left(n^{3(r+1) /(2 r)}\right)$ uniformly for $-1 \leqq t \leqq 1$ and for $x$ in any closed interval interior to $(-1,1)$. The relation (9) becomes

$$
\int_{-1}^{1} \rho(t)\left|H_{n}(x, t)\right|^{\gamma} d t=O\left(n^{(3 \gamma-4)(r+1) /(2 r)}\right),
$$

with the understanding that $\gamma>2$, as before, and (10) is to be replaced by

$$
\left|\int_{-1}^{1} \rho(t) \phi(t) H_{n}(x, t) d t\right|=O\left(n^{(4-\alpha)(r+1) /(2 \alpha r)}\right),
$$

the exponent which previously appeared in the right-hand member being multiplied by $(r+1) / r$ in each case. The same order of magnitude is found if $H_{n}(t, x)$ is substituted for $H_{n}(x, t)$. The exponent in (11) is replaced by

$$
\frac{(4-\alpha)(r+1)}{2 \alpha r}-1
$$

which is negative if $\alpha>(4 r+4) /(3 r+1)$. The last fraction is greater than 1 for any positive $r$, and is less than 2 by virtue of the hypothesis that $r>1$; it is in fact greater than the old value $4 / 3$, which it approaches when $r$ is very large.

It thus becomes clear how the conditions for convergence at the end of the preceding section are to be modified. The greater generality of the weight function is compensated by a closer

* See this Bulletin, loc. cit., p. 894, Theorem 4. 
restriction on the difference quotient $\phi(t)$, to the extent that the lower bound $4 / 3$ for $\alpha$ is increased to $(4 r+4) /(3 r+1)$. The corresponding lower bound for $\beta$ in (12) is $(r+3) /(4 r+4)=(1 / 4)$ $+1 /(2 r+2)$, instead of $1 / 4$. This is to be compared with the exponent $(r+1) /(2 r)=(1 / 2)+1 /(2 r)$ which appears in a theorem obtained elsewhere* for convergence of $s_{n}(x)$ toward $f(x)$.

5. More General Summation. Let the simple arithmetic mean which has been the subject of discussion hitherto be replaced by the more general expression

$$
\sigma_{n}(x)=\sum_{k=0}^{n-1} c_{n k} s_{k}(x),
$$

where the $c$ 's, within limits to be specified presently, are arbitrary constants satisfying the condition that

$$
\sum_{k=0}^{n-1} c_{n k}=1
$$

for each value of $n$. By virtue of the last condition,

$$
\sigma_{n}(x)-f(x)=\sum_{k=0}^{n-1} c_{n k}\left[s_{k}(x)-f(x)\right]
$$

For each $n$ let $A_{n}$ be the largest of the numbers $\left|c_{n k}\right|$. (Negative values for some of the $c$ 's are not excluded.) Repetition of the reasoning of $\$ 2$, with obvious modifications in detail, shows that if $\rho \phi^{2}$ is summable and $\rho$ has a positive lower bound,

$$
\left|\sigma_{n}(x)-f(x)\right|=O\left(n^{1 / 2} A_{n}\right)
$$

Convergence will take place if $\lim _{n \rightarrow \infty} n^{1 / 2} A_{n}=0$. Specifications with regard to uniformity of convergence are the same as before. It is only superficially a further generalization if $\sum c_{n k}$, instead of being equal to 1 for each $n$, is merely required to approach 1 as a limit. For if $\sum c_{n k}=e_{n}$ and $e_{n} \rightarrow 1$ the argument just outlined can be applied to $\sigma_{n}(x) / e_{n}$, and this expression and $\sigma_{n}(x)$ converge simultaneously.

* This Bulletin, loc. cit., p. 906, Theorem 16, $m=2$. The difference in exponents in directly significant, in spite of the fact that it is accompanied by other differences in substance and form of statement. 
A detailed working out of the results of combining the principle of this section with those of $\S \S 3$ and 4 may be omitted.

6. Trigonometric Approximation. The methods used above can be applied similarly to problems of the summation of series of orthogonal trigonometric sums.* As frequently occurs in such parallel developments, the work is in some respects simpler in the trigonometric problem and in other respects more complicated. By reason of the fact that in one of the lemmas employed a factor $n^{2}$ in the polynomial case is replaced by $n$ in the case of trigonometric sums, $\uparrow$ the condition $\alpha>4 / 3$ in $\S 3$ is replaced by the simpler condition $\alpha>1$, and the condition $\beta>1 / 4$ by $\beta>0$. The corresponding requirements in $\S 4$ are $\alpha>1+(1 / r)$ and $\beta>1 /(r+1)$.

Analogous results can be obtained for the case of polynomial approximation if $\rho(t)$ becomes infinite in a suitable manner at the ends of the interval. $\ddagger$

The University of Minnesota

* See D. Jackson, Orthogonal trigonometric sums, Annals of Mathematics, (2), vol. 34 (1933), pp. 799-814.

† See this Bulletin, loc. cit., p. 891, Lemma 1, as compared with Lemma 2, previously cited.

$\ddagger$ See this Bulletin, loc. cit., pp. 895-896, §4. 